\title{
VALIDEZ DEL PARCIAL DE ORINA Y EL GRAM EN EL DIAGNÓSTICO DE INFECCIÓN DEL TRACTO URINARIO EN EL EMBARAZO. HOSPITAL SIMÓN BOLÍVAR, BOGOTÁ, COLOMBIA, 2009-2010
}

\section{Validiity of uninalysis and Gram staining in the diagnosis of uninary tract infections in pregnancy. Hospital Simón. Bollivar, Bogotá, Colombia, 2009-2010}

Andrés Reyes-Hurtado, $M D^{1}$; Ana Gómez-Ríos, $M^{2} D^{2}$;orge A. Rodríguez-Ortiz, $M D^{3}$

Recibido: junio 20/12 - Aceptado: marzo 22/13

\section{RESUMEN}

Objetivo: determinar la exactitud diagnóstica del parcial de orina y la tinción de Gram en el diagnóstico de infección del tracto urinario (ITU) en el embarazo.

Materiales y métodos: estudio de validez diagnóstica en pacientes embarazadas hospitalizadas, con alta sospecha de ITU, en el servicio de Ginecología y Obstetricia del Hospital Simón Bolívar, Centro de III Nivel de atención, entre el 1 de enero de 2009 y el 31 de diciembre de 2010. El parcial de orina, sus parámetros - estearasa leucocitaria, bacteriuria, leucocituria, nitritos-y la tinción de Gram se compararon con el urocultivo considerado como patrón de oro diagnóstico. Se llevó a cabo un muestreo no probabilístico por conveniencia. El cálculo de tamaño de muestra se realizó mediante Epidat 4.0 con sensibilidad mínima esperada del 80\%, es-

Ginecoobstetra, Universidad El Bosque. Bogotá, Colombia

Residente IV año Ginecología y Obstetricia, Universidad El Bosque. Bogotá, Colombia.

3 Ginecoobstetra. Epidemiólogo Clínico, Hospital Simón Bolívar. Profesor asociado Universidad El Bosque. Docente Universidad de La Sabana. Bogotá, Colombia. jaurodriguez@unbosque.edu.co pecificidad mínima esperada del $90 \%$ y un nivel de confianza del $90 \%$ para la prevalencia establecida en la literatura del 20\%. Se calculó la sensibilidad, la especificidad, el valor predictivo positivo (VPP), valor predictivo negativo (VPN), además de la razón de probabilidades ( $\mathrm{RP}+\mathrm{y} \mathrm{RP}-$ ).

Resultados: un total de 212 muestras de pacientes embarazadas fueron incluidas en el estudio de las cuales 115 (54\%) presentaron urocultivo positivo y 97 (46\%) negativo. El 78\% de las pacientes presentaron pielonefritis, mientras el 22\% se presentaron con ITU baja (cistouretritis). El principal germen encontrado fue la Escherichia coli en el 70\% de las pacientes. El Gram tuvo una sensibilidad del 74\% (IC 95\%: 65-81) y una especificidad del 86\% (IC 95\%: 78-92), el parcial de orina mostró una sensibilidad del 21\% (IC 95\%: 14-29) y una especificidad de 92\% (IC 95\%: 84-96).

Conclusión: las pruebas rápidas que se realizan en el servicio de urgencias fueron altamente específicas en el diagnóstico de ITU en pacientes embarazadas. El Gram es la prueba con mayor sensibilidad para el diagnóstico de ITU en las pacientes gestantes del Hospital Simón Bolívar. 
Palabras clave: infecciones urinarias, diagnóstico, embarazo.

\section{ABSTRACT}

Objective: To determine the diagnostic accuracy of urinalysis and Gram staining the diagnosis of urinary tract infection (UTI) in pregnancy.

Materials and methods: Diagnostic validity study in pregnant women with a high suspicion of UTI, hospitalized in the Obstetrics and Gynecology Service of the Simon Bolivar Hospital, a Level III center, between January 1st 2009 and December 31st 2010. Gram staining and urinalysis with its parameters (leukocyte esterase, bacteriuria, leukocyturia, nitrites) were compared with urine culture, considered as the gold standard test. Specificity, sensitivity, positive predictive value (PPV) and probability ratios (LR + and PR -) were calculated. A non-probabilistic sampling was done out of convenience. The sample size was calculated using the Epidat 4.0, with a minimum expected sensitivity of $90 \%$ and a confidence level of $90 \%$ for the prevalence of $20 \%$ established in the literature. Results: Of the total of 212 samples of pregnant women included in the study, 115 (54\%) had a positive urine culture and 97 (46\%) had a negative culture. Seventy-eight per cent of patients presented with pyelonephritis, while $22 \%$ presented with low UTI (cystourethritis). The most frequent germ was Escherichia coli, found in $70 \%$ of patients. Gram stain had a sensitivity of 74\% (95\% CI: 65-81) and a specificity of $86 \%$ (95\% CI: 78-92); urinalysis had a sensitivity of $21 \%$ (95\% CI: 14-29) and a specificity of 92\% (95\% CI: 84-96).

Conclusion: Rapid tests done in the emergency room showed high specificity for the diagnosis of UTI in pregnant women. Gram staining is the test with the highest sensitivity for the diagnosis of UTI in this population at Hospital Simón Bolívar.

Key words: Urinary tract infections, diagnosis, pregnancy.

\section{INTRODUCCIÓN}

La infección del tracto urinario (ITU) en el embarazo se define como toda invasión microbiana del aparato urinario (riñón, uréteres, vejiga, uretra) que sobrepasa la capacidad de defensa del individuo afectado. Está clasificada como baja (bacteriuria asintomática, cistitis) y alta (pielonefritis). La importancia de las dos primeras es que pueden conducir a la infección urinaria alta en el embarazo y la última puede producir consecuencias graves como ruptura prematura de membranas (RPM) o parto pretérmino (1). Es la patología infecciosa bacteriana más común en el embarazo encontrándose hasta en el 20\% de las pacientes. Los agentes etiológicos asociados con bacteriuria son similares en las mujeres embarazadas y no embarazadas (1-5). La vía más común de ingreso de uropatógenos en el tracto urinario es la ascendente; las bacterias acceden a través de la uretra, ascienden a la vejiga y pueden llegar hasta el sistema colector (4, 6). La Escherichia coli sigue siendo responsable de 75 a 95\% de los casos sin complicaciones (6-9). El S. saprophyticus se ha aislado en el 5 a 15\% de las infecciones urinarias, y los enterococos Klebsiella y Proteus mirabilis en el restante 5 al 10\%. La infección por Staphylococcus saprophyticus, un organismo agresivo adquirido en la comunidad, puede también causar infección del tracto urinario superior, presentando con mayor frecuencia infecciones recurrentes o persistentes (10).

Las ITU ocurren con mayor frecuencia en las mujeres embarazadas por motivos fisiológicos (glucosuria, aminoaciduria,), mecánicos (compresión uterina, hidronefrosis) y hormonales (progesterona) que contribuyen al proceso natural de la enfermedad durante la gestación. También se han asociado el aumento del pH, incremento en la tasa de filtración glomerular (TFG) y la producción de orina, aumento del reflujo vesicoureteral, la menor capacidad de defensa del epitelio del aparato urinario bajo, el incremento de la secreción urinaria de estrógenos y el ambiente hipertónico de la médula renal (11-16). 
Existen varios métodos diagnósticos para la detección de ITU en el embarazo, sin embargo, el urocultivo sigue siendo la prueba de oro considerándolo como positivo cuando se encuentran más de 100000 unidades formadoras de colonias (UFC) $(17,18)$. La orina usualmente es estéril y la sola presencia de gérmenes constituye la posibilidad de un proceso infeccioso (en una muestra adecuadamente tomada y procesada en tiempo prudencial) o indica la contaminación de la muestra. Infortunadamente, el urocultivo es un examen que tarda entre 24 y 48 horas en arrojar resultados después de iniciar la incubación de la muestra (19). Por esta razón el parcial de orina y la tinción de Gram podrían presentarse como una herramienta rápida y confiable que nos permitiría analizar la posibilidad de ITU en el servicio de urgencias $(20,21)$.

La relevancia de utilizar métodos confiables y rápidos de diagnóstico de ITU radica en la importancia de iniciar oportunamente el manejo antibiótico, si se requiere, disminuyendo de esta manera la morbilidad materno perinatal y sus complicaciones asociadas tales como aborto, infección intraamniótica, parto pretérmino, preeclampsia, anemia, RCIU, RPM y sepsis materna o neonatal (16-18).

La exactitud de los métodos para el diagnóstico de la ITU puede variar según el tipo de institución, el tipo de ITU y los medios utilizados para el cultivo, por lo que es muy importante conocer el desempeño diagnóstico de los métodos utilizados como criterio de tratamiento inicial en las instituciones que atienden pacientes obstétricas, información que no ha sido evaluada en el Hospital Simón Bolívar (17). El objetivo del presente estudio es determinar la validez y efectividad del parcial de orina y la tinción de Gram en el diagnóstico de infección urinaria durante el embarazo, teniendo como referencia el urocultivo.

\section{MATERIALES Y MÉTODOS}

Estudio de validez de prueba diagnóstica en pacientes embarazadas hospitalizadas por alta sospecha de ITU en la Unidad de Alto Riesgo Obstétrico del servicio de Ginecología y Obstetricia del Hospital Simón Bolívar (III nivel de atención) de Bogotá, Colombia, que atiende pacientes del régimen de aseguramiento subsidiado por el Estado y bajo nivel socioeconómico, entre el 1 de enero de 2009 y el 31 de diciembre de 2010. A las pacientes con sintomatología como disuria, poliaquiuria, tenesmo vesical con o sin dolor pélvico se les tomó parcial de orina y tinción de Gram, y posteriormente urocultivo para el diagnóstico. Se incluyeron todas las pacientes independientemente de la edad gestacional, exceptuando las que usaron alguna terapia antibiótica en los últimos siete días y a las que no se les realizó urocultivo o este último mostró contaminación. Se realizó un muestreo no probabilístico por conveniencia. El cálculo de tamaño de muestra se realizó mediante Epidat 4.0 con sensibilidad mínima esperada del 80\%, especificidad mínima esperada del 90\% y un nivel de confianza del $90 \%$ para la prevalencia establecida en la literatura del 20\%.

Todas la muestras de orina para la realización de parcial de orina, Gram y cultivo fueron obtenidas con previo aseo vaginal de la paciente, orina espontánea y del chorro medio, con un volumen de 10 a $15 \mathrm{ml}$ en el tubo correspondiente. El Gram se procesó en orina sin centrifugar. El examen fisicoquímico de la muestra se realizó previa centrifugación por $10 \mathrm{~min}$ a $4000 \mathrm{rpm}$. El procesamiento de las muestras fue realizado por el personal de laboratorio clínico y el reporte del urocultivo se llevó a cabo sin conocimiento previo del resultado del parcial de orina o de la tinción de Gram. El resultado de más de 100.000 UFC en el urocultivo se consideró diagnóstico de infección urinaria. Los parámetros en el parcial de orina que se tomaron como de alta sospecha para infección urinaria fueron más de cinco leucocitos por campo de alto poder, más de cinco glóbulos rojos, o más $2+$ de bacterias. Asimismo, un Gram de orina con microorganismos presentes en cualquier número tipo bacilos Gram-negativos o cocos Gram-positivos se consideró indicativo de infección urinaria.

Las variables estudiadas fueron edad materna, edad gestacional, tipo de infección urinaria, germen 
aislado en urocultivo y su resistencia antibiótica, reporte del Gram, y los parámetros del parcial de orina como bacteriuria, $\mathrm{pH}$, leucocituria nitritos y estearasa leucocitaria.

La información obtenida en un instrumento propio se digitó en base de datos de Excel y posteriormente se realizó análisis estadístico en Epidat 4.0, realizando tablas 2 × 2 para cada paraclínico y formulando pruebas de sensibilidad, especificidad, valor predictivo positivo (VPP), valor predictivo negativo (VPN) y razón de probabilidades (RP). El estudio fue aprobado por el comité de ética del Hospital Simón Bolívar.

\section{RESULTADOS}

De las 212 pacientes que fueron hospitalizadas en el servicio de alto riesgo obstétrico y cumplieron con todos los criterios de inclusión para el estudio, 115 (54\%) presentaron infección urinaria por urocultivo positivo y las restantes 97 (46\%) tuvieron resultado negativo. Del grupo de pacientes con urocultivo positivo, 90 (78\%) se presentaron con cistouretritis y 25 (22\%) con pielonefritis. La edad de las pacientes osciló entre los 14 y los 42 años con una media de 23 años. La edad gestacional estratificada por trimestre presentó una frecuencia de 30\% en el primer trimestre, $43 \%$ en el segundo trimestre y $27 \%$ en el tercer trimestre. El germen más frecuentemente aislado fue la Escherichia coli en 81 pacientes que representa el 70\% del total, seguido de la Klebsiella Pneumoniae en 9 pacientes (8\%), Enterococcus faecalis en 8 (7\%), Staphylococcus coagulasa negativo en 8 (7\%), Proteus mirabilis en 5 (4\%), Serratia marcescens en 2 (2\%), Estreptococo del grupo B en una paciente y Pseudomonas aeruginosa en una paciente.

Dentro de los paraclínicos analizados se encontró que la sensibilidad del Gram en el diagnóstico de ITU fue de 74\% (IC 95\%: 65-81), la especificidad del 86\% (IC 95\%: 78-92), con un VPP de 87\% y un VPN de 74\%. Respecto al parcial de orina la sensibilidad fue del 21\% (IC 95\%: 14-29), especificidad de 92\% (IC 95\%: 84-96) con VPP de 75\% y VPN de 49\%. En cuanto a los parámetros del parcial de orina se encontró que la estearasa leucocitaria fue más sensible que los nitritos en la detección de un parcial de orina patológico que sugiera posteriormente infección del tracto urinario (66\% frente a 30\%). La especificidad de los nitritos fue del $97 \%$ en comparación con el 74\% de la estearasa leucocitaria. Los parámetros más sensibles dentro del parcial de orina fueron la bacteriuria y la leucocituria (tabla 1). La razón de probabilidades $\mathrm{RP}+$ para el parcial de orina fue de 2,5 y el RP - fue de 0,86. Para el Gram fue de 5,28 y de 0,30 respectivamente.

\section{DISCUSIÓN}

En este periodo, de un total de 1019 pacientes que presentaron diagnóstico de ITU se hospitalizaron 212 en el periodo de enero de 2009 a diciembre de 2010. En el grupo de pacientes hospitalizadas se tuvo una prevalencia de infección urinaria confirmada del 54\%. Del grupo de pacientes con urocultivo positivo, 90 (78\%) se presentaron con cistouretritis y 25 (22\%) se diagnosticaron como pielonefritis. En los servicios de urgencias se evidencia una tendencia a subdiagnosticar de manera clínica o por parcial de orina la ITU. Ya ha sido descrito que los hallazgos clínicos sugestivos son muy poco sensibles para predecir la enfermedad $(17,22)$.

La población estudiada se encontró entre los 14 a 42 años con un promedio de edad de 23 años, que coincide con los hallazgos de los estudios en la población latinoamericana (23). El tiempo de gestación en el que más se presentó ITU en nuestro estudio fue en el segundo trimestre con una frecuencia de $43 \%$, diferente a la mayoría de los estudios referidos en embarazadas en los cuales las infecciones urinarias se presentan con mayor frecuencia en el tercer trimestre de gestación (9).

En la literatura, de los paraclínicos considerados el uroanálisis tiene en la mayoría de estudios analizados una sensibilidad aproximada del 65,4\% y especificidad del 74,4\%, lo cual varía de forma importante con nuestro estudio $(9,24)$; sin embargo, en una investigación realizada en la Clínica Mayo en pacientes gestantes se hicieron hallazgos muy 


\begin{tabular}{|c|c|c|c|c|c|c|c|c|c|c|}
\hline Prueba & VP & FP & FN & VN & $\begin{array}{l}\text { SENS } \\
(\%)\end{array}$ & $\begin{array}{c}\text { ESPEC } \\
(\%)\end{array}$ & $\begin{array}{l}\text { VPN } \\
(\%)\end{array}$ & $\begin{array}{l}\text { VPP } \\
(\%)\end{array}$ & $\mathbf{R P}+$ & $\mathbf{R P}$ - \\
\hline Gram & 85 & 13 & 30 & 84 & 74 & 86 & 74 & 87 & 5,28 & 0,30 \\
\hline P. de O. & 24 & 8 & 91 & 89 & 21 & 92 & 49 & 75 & 2,5 & $\mathrm{O}, 86$ \\
\hline $\begin{array}{l}\text { Estearasa } \\
\text { leucocitaria }\end{array}$ & 76 & 25 & 39 & 72 & 66 & 74 & 64 & 75 & 2,5 & 0,45 \\
\hline Nitritos & 35 & 3 & 80 & 94 & 30 & 97 & 54 & 92 & 10 & 0,72 \\
\hline Leucocituria & 84 & 28 & 31 & 69 & 73 & 71 & 69 & 75 & 2,5 & 0,38 \\
\hline Hematuria & 25 & 19 & 90 & 78 & 21 & 80 & 46 & 56 & 1,05 & 0,98 \\
\hline Bacteriuria & 84 & 14 & 31 & 83 & 73 & 85 & 72 & 85 & 4,86 & 0,31 \\
\hline
\end{tabular}

semejantes a los encontrados en este estudio, con una sensibilidad para el uroanálisis del 22\% y una especificidad del 99\% (17).

Los nitritos tienen resultados altamente heterogéneos en la literatura con sensibilidad del 37-67\% y especificidad del $85-98 \%$, lo cual es semejante a lo referido en nuestro estudio, por lo que la ausencia de este parámetro nunca se descarta en el diagnóstico de ITU, aunque su presencia casi que la confirma (19-21). Adicional a lo anterior, se ha informado que la esterasa leucocitaria tiene una sensibilidad del 50-70\% y especificidad del 92-95\% (19-21, 24). La suma de las dos pruebas para el diagnóstico de ITU: estearasa leucocitaria más nitritos, alcanza una sensibilidad del 93\% y una especificidad del $72 \%(23,25)$.

La presencia de leucocituria tiene una sensibilidad global del 32\%, muy disminuida con respecto a la relacionada en el presente estudio, y una especificidad del 92\%. La tinción de Gram en orina sin centrifugar tiene una sensibilidad de 86-94\% y una especificidad del 89-100\% para diagnóstico de ITU, lo cual es ligeramente superior a lo encontrado en nuestro estudio (17, 19-21).

Las bacterias Gram negativas siguen ocupando los primeros lugares en frecuencia en cuanto a etiología, siendo la Escherichia coli el principal germen (70\% de los casos), seguido de los enterococos y los staphylococos (principalmente los coagulasa negativos) como lo comprueba nuestro estudio, y es similar a estudios previos realizados en múltiples y variados países. La alta frecuencia de infección urinaria por E. coli podría estar relacionada a la estasis urinaria fisiológica y a la pobre higiene genital de nuestras pacientes, con el agravante de alta tasa de resistencia antibiótica y tratamientos incompletos que se inician sin conocimiento del germen causal $(26,27)$.

En un servicio de urgencias obstétricas se deben buscar pruebas diagnósticas en orina que incrementen la probabilidad de detectar las pacientes verdaderamente enfermas (alta sensibilidad), las pruebas en el Hospital Simón Bolívar tienen una alta tasa de falsos negativos, aunque la tasa de falsos positivos es baja.

\section{CONCLUSIÓN}

El Gram de orina, con una sensibilidad de 74\%, es la prueba más útil para el diagnóstico de ITU en embarazo en el Hospital Simón Bolívar. El parcial de orina presenta una baja sensibilidad. Ambas pruebas tienen una baja tasa de falsos positivos (14 y $8 \%$ respectivamente) de manera que se evitan tratamientos innecesarios que tienen el potencial de generar resistencia bacteriana. 


\section{REFERENCIAS}

1. Ferreira FE, Olaya SX, Zúñiga P, Angulo M. Infección urinaria durante el embarazo, perfil de resistencia bacteriana al tratamiento en el Hospital General de Neiva, Colombia. Rev Colomb Obstet Ginecol 2005;56:239-43.

2. Schnarr J, Smaill F. Asymptomatic Bacteriuria and Symptomatic Urinary Tract Infections In Pregnancy. Eur J Clin Invest 2008;38:S50-7.

3. Hamdan HZ, Ziad AH, Ali SK, Adam I. Epidemiology of Urinary Tract Infections and Antibiotics Sensitivity Among Pregnant Women At Khartoum North Hospital. Ann Clin Microbiol Antimicrob 2011;10:2.

4. Macejko AM, Schaeffer AJ. Asymptomatic Bacteriuria and Symptomatic Urinary Tract Infections During Pregnancy. Urol Clin North Am 2007;34:35-42.

5. Florentin L, Lascurain A, Báez D, Martínez M, Troche A. Consenso de infección urinaria: pautas de diagnóstico y tratamiento. Pediatr (Asunción) 2011;38:68-72.

6. Abarzúa F, Zajer C, Donoso B, Belmar C, Riveros JP, González P, et al. Reevaluación de la sensibilidad antimicrobiana de patógenos urinarios en el embarazo. Rev Chil Obstet Ginecol 2002;67:226-31.

7. Alvarez G, Echeverría J, Garau A, Lens V. Infección Urinaria y embarazo. Diagnóstico y terapéutica. Rev posgrado VIa Cátedra Med 2006;150:20-3.

8. Pino T, Sabina A. Evaluación de la prescripción de gentamicina en gestantes ingresadas con infección del tracto urinario. Rev Cubana Obstet Ginecol 2005;31.

9. Norris DL, Young JD. Urinary Tract Infections: Diagnosis And Management In The Emergency Department. Emerg Med Clin North Am 2008;26:413-30.

10. Gupta K, Hooton TM, Stamm WE. Increasing Antimicrobial Resistance and the Management of Uncomplicated Community-Acquired Urinary Tract Infections. Ann Intern Med 2001;135:41-50.

11. Arroyave V, Cardona A, Castaño JJ, Giraldo V, Jaramillo M, Moncada N, et al. Caracterización de la infección de vías urinarias en mujeres embarazadas atendidas en una unidad de primer nivel de atención (Manizales, Colombia), 2006-2010. Arch Med (Manizales) 2011;11:39-50
12. Mittal $P$, Wing DA. Urinary Tract Infections in Pregnancy. Clin Perinatol 2005;32:749-64.

13. Ovalle A, Levancini M. Urinary Tract Infections in Pregnancy. Curr Opin Urol 2001;11:55-9.

14. Shrotri KN, Morrison ID, Shrotri NC. Urological Conditions in Pregnancy: A Diagnostic and Therapeutic Challenge. J Obstet Gynaecol 2007;27:648-54.

15. Thomas AA, Thomas AZ, Campbell SC, Palmer JS. Urologic Emergencies in Pregnancy. Urology 2010;76:453-60.

16. Johnson EK, Kim ED. Urinary Tract Infections in Pregnancy [en línea] 2009. Visitado 2012 Abr 1. Disponible en: http://Emedicine.Medscape.Com/Article/452604 Overview\#Aw2aab6b4

17. Bachman JW, Heise RH, Naessens JM, Timmerman MG. A study of various tests to detect asymptomatic urinary tract infections in an obstetric population. JAMA 1993;270:1971-4.

18. Mandell D. Bennett's Principles and Practice of Infectious Diseases, $7^{\text {th }} \mathrm{Ed}$. Urinary Tract Infection In Pregnancy [en línea] 2009. Visitado 2012 Abr 1. Disponible en: http:/Www.Mdconsult.Com/Books/ Page.Do?Sid=0\&Eid=4-U1.0-B978-0-44

19. Mustafa Y, Idris S. The validity of the rapidly diagnostic tests for early detection of urinary tract infection. Düzce Medical Journal 2008;3:39-42.

20. Devillé WL, Yzermans JC, van Duijn NP, Bezemer PD, van der Windt DA, Bouter LM. The urine dipstick test useful to rule out infections. A meta-analysis of the accuracy. BMC Urol 2004;4:4.

21. Kacmaz B, Cakir O, Aksoy A, Biri A. Evaluation of rapid urine screening test to detect asymptomatic bacteriuria in pregnancy. Jpn J Infect Dis 2006;59:261-3.

22. Lammers RL, Gibson S, Kovacs D, Sears W, Strachan G. Comparison of Test Characteristics of Urine Dipstick and Urinalysis at Various Test Cutoff Points. Ann Emerg Med 2001;38:505-12.

23. Estrada-Altamirano A, Figueroa-Damián R, VillagranaZesati R. Infección de vías urinarias en la mujer embarazada. Importancia del escrutinio de bacteriuria asintomática durante la gestación. Perinatol Reprod Hum 2010;24:182-6.

24. Feitosa DC, da Silva MG, de Lima Parada CM. Accuracy of simple urine tests for diagnosis of urinary 
tract infections in low-risk pregnant women. Rev Lat Am Enfermagem 2009;17:507-13.

25. Nicolle L, Bradley S, Colgan R, Rice J, Schaeffer A, Hooton T. Infectious Diseases Society of America guidelines for the diagnosis and treatment of asymptomatic bacteriuria in adults. Clin Infect Dis 2005;40:643-54.
26. Demilie T, Beyene G, Melaku S, Tsegaye W. Urinary bacterial profile and antibiotic susceptibility pattern among pregnant women in North West Ethiopia. Ethiop J Health Sci 2012;22:121-8.

27. Casas RL, Ortiz M, Erazo-Bucheli D. Prevalencia de la resistencia a la ampicilina en gestantes con infección urinaria en el Hospital Universitario San José de Popayán (Colombia) 2007-2008. Rev Colomb Obstet Ginecol 2009;60:334-8. 